Among 4156 RTs conducted, 626(15\%) were positive for syphilis. The characteristics of the population tested were: 2003(48\%) women, 374(9\%) homo/bisexual, 710(17\%) under 25 years and 1001(24\%) elderly. In the subgroup with positive syphilis RTs, 250(39.9\%) were women, 29(4.9\%) homo/ bisexual, 44(7\%) under 25 years, 43(6.8\%) positive RT for HIV and 43(6.8\%) for Hepatitis C. Although the RTs for syphilis did not confirm diagnosis, the framework in place to deal with positive tests provides same-day medical consultation to evaluate the need for immediate treatment at the event site and referral for follow-up or continued treatment at the Primary Healthcare level.

Conclusion Social interaction with the public during RT using a mobile healthcare units in places intended for leisure sought to increase awareness and access to people with undiagnosed STIs. Ensuring that specific subgroups of the general population have access to testing and medical consultation were shown to be important points when applying point of care tests. These aspects of community testing should be evaluated in future research.

Disclosure No significant relationships.

\section{P281 REACHING OUT TO MSM WHO FLED FOR WAR AND LGBT OPPRESSION FOR HIV AND STI TESTING, HBV VACCINATION AND COUNSELLING}

${ }^{1}$ Wim Zuilhof, ${ }^{2}$ Hanna Bos*. ${ }^{1}$ SOA AIDS Nederland, MSM Programme, Amsterdam, Netherlands; ${ }^{2}$ SOA AIDS Nederland, Amsterdam, Netherlands

\subsection{6/sextrans-2019-sti.406}

Background The Netherlands has had a large influx of MSM refugees in recent years. Soon after arriving many of them start dating other MSM for sexual hook-ups, while insufficiently aware of risks that sex between men entails for HIV/ STI. They are also unfamiliar with existing facilities for HIV/ STI testing, HBV-vaccination and HIV-care in the Netherlands. Methods In 2017 and 2018, MSM refugees were invited for meet-ups in Amsterdam and three other cities, where information and counseling about STI and HIV and how to prevent these was given in multiple languages. HIV and STI tests and vaccination for $\mathrm{HBV}$ were also offered on the spot. Only refugees were allowed in, thus creating a safer space atmosphere. Those tested and/or vaccinated received follow-up appointments on regular office hours for test results, further HIV/STI care or completion of HBV vaccinations. These meet-ups were possible through very close cooperation between self-organizations of migrant MSM, regional Public Health Services, gay bars and a national NGO.

Results In 2017 and 2018 a total of 858 MSM refugees attended 8 meet-ups on which $223 \mathrm{HIV} / \mathrm{STI}$ tests were done and $226 \mathrm{HBV}$-vaccinations were given. 43 STI's and $3 \mathrm{HIV}$ infections were found. A survey among visitors showed huge appreciation of the meet-ups. A large proportion of the visitors visited more than one meet up, thus showing appreciation for and trust in the organisers. One on one counselling of visitors by volunteers and health professionals during the meet ups showed lack of knowledge about HIV and STI and lack of behavioural skills among visitors and the need for development of behavioural interventions specifically for this group. Conclusion MSM refugees can be reached through gay community meet-ups for HIV/STI testing and HBV vaccination. Extra data have to be collected for the development of much needed behavioural interventions.

Disclosure No significant relationships.

\section{P283 THE ALBERTA 2016-2020 STBBI OPERATIONAL STRATEGY AND ACTION PLAN: COLLECTIVE IMPACT TO ADDRESS THE PROVINCIAL STI OUTBREAK}

${ }^{1}$ Cari Egan*, ${ }^{1}$ Christopher Wood, 'David Strong, ${ }^{2}$ Jennifer Gratrix, ${ }^{2}$ Petra Smyczek, ${ }^{3}$ Abhaya Prasad, ${ }^{3}$ Lindsay Rathjen. ${ }^{1}$ Alberta Health Services, Communicable Disease Control, Calgary, Canada; ${ }^{2}$ Alberta Health Services, STI Services, Edmonton, Canada; ${ }^{3}$ Alberta Health Services, Edmonton, Canada

\subsection{6/sextrans-2019-sti.407}

Background In April of 2016, a provincial sexually transmitted infections (STI) outbreak was declared in Alberta. In response to sharply rising rates, Alberta formed the Alberta Sexually Transmitted and Blood Borne Infection (STBBI) Network (ASN) and developed the 2016-2020 STBBI Operational Strategy and Action Plan (OSAP). The aim of the STBBI OSAP is to transform STBBI care in Alberta by leveraging engagement and commitment from within the ASN to achieve collective impact and develop wrap around shared care models through formalized interagency collaboration.

Methods ASN has grown to include over 500 stakeholders representing approximately 200 community-based, provincial and federal organizations including communities most affected by STBBI (Indigenous, LGBTQ2+, immigrants, and street involved clients). The OSAP comprises 74 recommendations that focus on five pillars of client-centred STBBI care: (1) prevention, (2) testing/screening, (3) treatment, (4) linkage to care, and (5) stigma. Seven working groups designed 15 project concepts with endorsement from the Steering Committee to optimize achievement of prioritized recommendations.

Results Implementation of STBBI OSAP priority recommendations is still underway in 2019. Some prioritized action items include: PrEP guidelines and training program for designated prescribers; a validation study of self-collected gonorrhea/chlamydia NAAT specimens to improve access to low barrier testing opportunities; expedited partner therapy; expanded Hepatitis C treatment access in Indigenous communities; education for primary care providers to increase access and linkage to STBBI care; and formalizing interagency collaboration through the development of STBBI wrap around shared care model demonstration projects.

Conclusion The STBBI OSAP is an innovative, comprehensive, and collaborative intersectoral approach to address the high burden of STBBI in Alberta and to strengthen the provincial outbreak response. The strength of this provincial initiative is the engagement and collaboration amongst ASN stakeholders to achieve long lasting impact and sustainability of both systemic and operational interventions.

Disclosure No significant relationships. 